\title{
Association of COVID-19 Outbreak with Changes in Physical Activity Among Adults with Elevated Risk for Major Adverse Cardiovascular Events
}

J Gen Intern Med 36(11):3625-8

DOI: $10.1007 / \mathrm{s} 11606-021-06725-5$

(C) This is a U.S. government work and not under copyright protection in the U.S.; foreign copyright protection may apply 2021

$\mathrm{W}$ hile the COVID-19 stay-at-home orders reduced the risk of viral transmission, their impact on preventive health behaviors of high-risk patients is unknown. Greater physical activity is associated with lower cardiovascular disease and mortality rates. ${ }^{1}$ For individuals at elevated risk for major adverse cardiovascular events (MACE), a short-term reduction in activity could impact longer-term habits. ${ }^{2}$ The objective of this study was to evaluate the association of the COVID-19 pandemic and the stay-at-home order issued on March 23, 2020, in Metropolitan Philadelphia with changes in physical activity among adults at elevated risk for MACE and examine differences in physical activity changes by sociodemographic characteristics.

\section{METHODS}

This study was approved by the University of Pennsylvania institutional review board and data was obtained from an ongoing randomized clinical trial evaluating the use of gamification and financial incentives to increase physical activity (NCT03911141). Participants were adults with either an atherosclerotic cardiovascular disease (ASCVD) condition or a 10 -year ASCVD risk score of $\geq 7.5 \%$ who used a Charge 3 (Fitbit) wearable to monitor activity. ${ }^{3}$

For each participant, daily steps and moderate-to-vigorous physical activity (MVPA) minutes were obtained. Daily MVPA minutes were estimated by totaling the number of minutes with a pace $\geq 100$ steps $/ \mathrm{min} .{ }^{4}$ We used multiple imputation for step values that were missing or $<1000 /$ day $^{5}$ ( $10.1 \%$ of data) and model results were pooled using Rubin's standard rules.

Using week 1 (February 1-7) of the pre-COVID-19 period (February 1-March 5, 2020) as reference, weekly changes in

Received October 18, 2020

Accepted March 15, 2021

Published online March 26, 2021 physical activity were estimated using a generalized linear mixed effects model adjusted for participant random effects, pre-trial baseline physical activity, study arm, and participant duration in the trial, in weeks. We then examined variations in physical activity by participant characteristics using a multivariate regression model that compared the period after Pennsylvania's first COVID-19 case (March 6-May 27, 2020) to the pre-COVID-19 period with interaction terms for time and characteristic. Analyses were conducted in R (version 3.6.1) using 2-sided hypothesis tests (significance level, $P$ $<.05)$.

\section{RESULTS}

The sample comprised 217 adults with mean (SD) age of 69 (6.6) years; $22.6 \%$ had an ASCVD condition, and $24.0 \%$ were city residents (Table 1). Among citydwelling participants, $21.1 \%$ were Black while $9.1 \%$ of suburban participants were Black. Participants had a pretrial baseline mean (SD) of 5483 (2039) steps and averaged 7257 (3378) steps during the pre-COVID-19 period (February 1-March 5, 2020). Adjusted results indicate daily steps significantly declined relative to week 1 of the preCOVID-19 period in the 2nd week after the state's first COVID-19 case ( -281 steps; $95 \% \mathrm{CI}-509,-55 ; P=.01$; Fig. 1). The greatest decline occurred in the first week after the stay-at-home order ( -617 steps; $95 \%$ CI - 887, - 346; $P<.001)$, but this change was no longer significantly different from week 1 by 4 weeks after the stay-at-home order (week 13). There were no sustained, significant changes in MVPA across the full sample (Fig. 1).

After adjusting for sociodemographic characteristics (Table 1), the largest, sustained changes in daily steps in the post-period, relative to the pre-period, were among Black vs. White participants $(-816 ; 95 \%$ CI -1039 , 594; $P<.001)$ and city vs. suburban residents $(-463,95 \%$ CI $-614,-311 ; P<.001)$. Each group also had significant, sustained declines in MVPA minutes (Table 1).

\section{DISCUSSION}

In a sample of adults at elevated risk for MACE, the COVID-19 pandemic and related stay-at-home order were 
Table 1 Sample Characteristics and Adjusted Changes in Physical Activity

\begin{tabular}{|c|c|c|c|c|c|}
\hline & \multirow[t]{2}{*}{ Participants $(n=217)$} & \multicolumn{2}{|c|}{$\begin{array}{l}\text { Adjusted change in daily steps } \\
\text { post-COVID-19 relative to pre- } \\
\text { period }\end{array}$} & \multicolumn{2}{|c|}{$\begin{array}{l}\text { Adjusted change in MVPA } \\
\text { minutes post-COVID-19 relative } \\
\text { to pre-period }\end{array}$} \\
\hline & & Estimate (95\% CI) & $P$ value & Estimate $(95 \% \mathrm{CI})$ & $P$ value \\
\hline \multicolumn{6}{|l|}{ Sociodemographics } \\
\hline Age, mean (SD), years* & $69(6.6)$ & $-3(-15,9)$ & 0.66 & $-0.2(-0.3,-0.1)$ & $<0.001$ \\
\hline \multicolumn{6}{|l|}{ Gender, no. $(\%)$} \\
\hline Male & $100(46.1)$ & Ref & N/A & Ref & N/A \\
\hline Female & $117(53.9)$ & $63(-76,203)$ & 0.37 & $0.3(-0.6,1.3)$ & 0.48 \\
\hline \multicolumn{6}{|l|}{ Race/ethnicity } \\
\hline White, non-Hispanic & $184(84.8)$ & Ref & N/A & Ref & N/A \\
\hline Black, non-Hispanic & $26(12.0)$ & $-816(-1039,-594)$ & $<0.001$ & $-3.5(-5.2,-1.8)$ & $<0.001$ \\
\hline Other & $7(3.2)$ & $-214(-586,157)$ & 0.26 & $-0.5(-2.7,1.7)$ & 0.66 \\
\hline \multicolumn{6}{|l|}{ Marital status, no. (\%) } \\
\hline Single & $23(10.6)$ & Ref & N/A & Ref & N/A \\
\hline Married & $140(64.5)$ & $-103(-326,120)$ & 0.36 & $-0.6(-2.1,0.9)$ & 0.41 \\
\hline Other & $54(24.9)$ & $20(-217,257)$ & 0.87 & $-1.2(-2.8,0.3)$ & 0.12 \\
\hline College graduate & $172(79.3)$ & $-276(-442,-110)$ & 0.001 & $-0.1(-1.2,1.0)$ & 0.84 \\
\hline \multicolumn{6}{|l|}{ Annual household income, no. (\%) } \\
\hline$<\$ 50,000$ & $45(20.7)$ & Ref & N/A & Ref & N/A \\
\hline$\$ 50,000$ to $\$ 100,000$ & $72(33.2)$ & $15(-177,207)$ & 0.88 & $-0.9(-2.2,0.5)$ & 0.20 \\
\hline$>\$ 100,000$ & $100(46.1)$ & $-83(-292,125)$ & 0.43 & $-0.8(-2.4,0.8)$ & 0.31 \\
\hline City residence, no. $(\%)$ & $52(24.0)$ & $-463(-614,-311)$ & $<0.001$ & $-2.6(-3.2,-1.3)$ & $<0.001$ \\
\hline \multicolumn{6}{|l|}{ Self-reported measures } \\
\hline \multicolumn{6}{|l|}{ Health status, no. (\%) } \\
\hline Excellent & $17(7.8)$ & Ref & N/A & Ref & N/A \\
\hline Very good & $86(39.6)$ & $-41(-286,204)$ & 0.74 & $-0.8(-2.3,0.7)$ & 0.31 \\
\hline Good ${ }^{\circ}$ & $91(41.9)$ & $9(-241,259)$ & 0.94 & $-1.7(-3.2,-0.1)$ & 0.04 \\
\hline Fair & $23(10.6)$ & $-24(-352,305)$ & 0.89 & $-1.7(-4.0,0.7)$ & 0.16 \\
\hline Poor & $0(0.0)$ & - & - & - & - \\
\hline Prior wearable device use, no. $(\%)$ & $146(67.3)$ & $-239(-365,-112)$ & $<0.001$ & $-1.0(-18,-0.1)$ & 0.03 \\
\hline \multicolumn{6}{|l|}{ Baseline measures } \\
\hline \multicolumn{6}{|l|}{ Existing ASCVD diagnosis, no. (\%) } \\
\hline No & $168(77.4)$ & Ref & Ref & Ref & Ref \\
\hline Yes & $49(22.6)$ & $-215(-372,-58)$ & 0.01 & $-0.1(-1.3,1.0)$ & 0.03 \\
\hline Body mass index, mean $(\mathrm{SD})^{\dagger}$ & $29.8(6.2)$ & $-23(-35,-12)$ & $<0.001$ & $0.0(-0.1,0.1)$ & 0.89 \\
\hline
\end{tabular}

SD, standard deviation; $C I$, confidence interval; MVPA, moderate-to-vigorous physical activity; ASCVD, atherosclerotic cardiovascular disease; Ref, referent group; N/A, not applicable

Multivariate generalized linear model adjusted for pre-trial baseline measure (daily steps or MVPA minutes), study arm, participant week in the clinical trial, each listed participant characteristic, time (before or after the first COVID-19 case on 3/6/20), and an interaction terms for time $\times$ participant characteristic. The table presents the estimate for the interaction terms

*Variable was grand mean centered. Estimate is for every 1 year increase above the mean value. Here, for every 1 year increase in age, participants took 3 fewer steps in the post-COVID period. For example, participants who were 70 years old took 3 fewer steps, and those who were 71 took 6 fewer steps

+ Variable was grand mean centered. Estimate is for every 1 point increase above the mean value. Here, for every 1 point increase in body mass index (BMI), participants took 23 fewer steps in the post-COVID period. For example, participants with a BMI of 30.8 (1 points above the mean) took 23 fewer steps in the post-COVID period, relative to the pre-COVID period

associated with a significant decline in daily steps that returned to pre-COVID-19 levels within 8 weeks. The insignificant change in MVPA minutes across the full sample suggests that, overall, individuals preserved higher intensity activities while sheltering in place but experienced a greater reduction in lighter activity. However, a significant, sustained decline in daily steps and MVPA was observed among participants with Black race and/or city residence over the 12-week post-period. These sustained reductions could impact long-term outcomes. Clinicians may consider increased monitoring or interven- tions to improve daily activity to prevent physical and cardiovascular decline while individuals are sheltering in place.

This study is limited in its observational design, geography, sample size, and shorter follow-up. The overall recovery period may be partially due to the trial intervention designed to improve physical activity. Our findings suggest the COVID19 pandemic could disproportionately reduce physical activity among minority and urban patients, further exacerbating existing disparities in preventive health behaviors. Longer term evaluations are needed. 

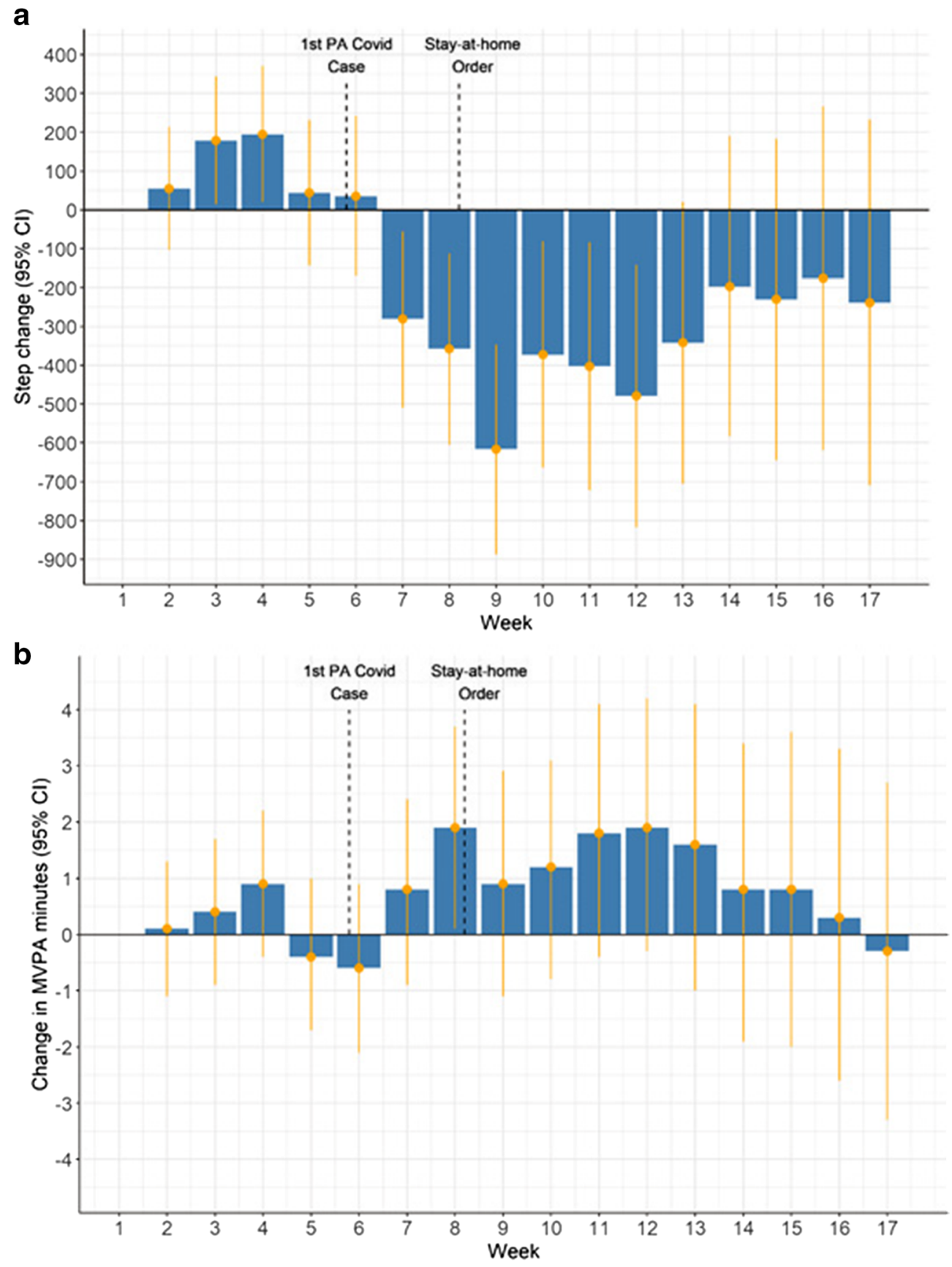

Fig. 1 Adjusted change in daily steps and MVPA minutes by week for the full sample. Adjusted change and $95 \%$ confidence intervals for daily steps (a) and MVPA minutes (b). The state's first COVID-19 case occurred on March 6, 2020, and the stay-at-home order was issued on March 23, 2020. The significant decline in daily steps began after the state's first COVID-19 case (week 7), with the greatest decline observed 1 week after the stay-at-home order (week 9). There were no significant, sustained changes in MVPA minutes across the full sample; however, participants who were Black and/or city-dwelling did experience sustained declines in MVPA (see Table 1). Physical activity is known to vary by season and the slight, but significant, increase in daily steps prior to the state's first COVID-19 case is likely due to seasonal changes.

Kimberly J. Waddell, $\mathrm{PhD}, \mathrm{MS}^{1,2}$

Kevin G. Volpp, $M D, P h D^{1,2,3,4,5}$

Neel P. Chokshi, MD, MBA ${ }^{1,3,5,6}$

Dylan S. Small, $P h D^{1,4,5}$

Louise B. Russell, $P h D^{3,5}$
Catherine Reale, $B S^{1}$

Mitesh S. Patel, MD, MBA ${ }^{1,2,3,4,5}$

${ }^{1}$ Penn Medicine Nudge Unit, University of Pennsylvania,

Philadelphia, PA, USA 
${ }^{2}$ Crescenz Veterans Affairs Medical Center,

Philadelphia, PA, USA

${ }^{3}$ Perelman School of Medicine, University of

Pennsylvania,

Philadelphia, PA, USA

${ }^{4}$ Wharton School, University of Pennsylvania,

Philadelphia, PA, USA

${ }^{5}$ LDI Center for Health Incentives and Behavioral

Economics, University of Pennsylvania,

Philadelphia, PA, USA

${ }^{6}$ Penn Center for Digital Cardiology, University of

Pennsylvania,

Philadelphia, PA, USA

Corresponding Author: Kimberly J. Waddell, PhD, MS; Penn Medicine Nudge Unit, University of Pennsylvania, Philadelphia, PA, USA (e-mail: Kimberly.Waddell@pennmedicine.upenn.edu).

Funding This study was supported by a grant from the National Heart, Lung, and Blood Institute (NIH R33HL141440) and the University of Pennsylvania Health System through the Penn Medicine Nudge Unit. Dr. Waddell was supported by the Department of Veterans Affairs Advanced Fellowship Program in HSR\&D. Dr. Patel was supported by a career development award from the Department of Veterans Affairs HSR\&D.

Data Availability Dr. Waddell had full access to all the data in the study and takes responsibility for the integrity of the data and the accuracy of the data analysis.

\section{Declarations:}

Conflict of Interest: Dr. Patel is the founder of Catalyst Health, a technology and behavior change consulting firm. Dr. Patel is an advisory board member for HealthMine Services Inc., LifeVest Health, and
Holistic Industries. Dr. Patel has also received research funding from Deloitte, which is not related to the work described in this manuscript. Dr. Volpp is a partner at VAL Health and has also received consulting income from CVS Caremark and research funding from Humana, CVS Caremark, Discovery (South Africa), Hawaii Medical Services Association, Oscar, and Merck, none of which are related to the work described in this manuscript. No other disclosures were reported.

Disclaimer: The funder had no role in the design and conduct of the study; collection, management, analysis, and interpretation of the data; preparation, review, or approval of the manuscript; and decision to submit the manuscript for publication.

\section{REFERENCES}

1. Yang Q, Cogswell ME, Flanders WD, et al. Trends in cardiovascular health metrics and associations with all-cause and CVD mortality among US adults. JAMA. 2012;307(12):1273-1283.

2. Hajat C, Hasan A, Subel S, Noach A. The impact of short-term incentives on physical activity in a UK behavioural incentives programme. NPJ Digit Med. 2019;2:91

3. Case MA, Burwick HA, Volpp KG, Patel MS. Accuracy of smartphone applications and wearable devices for tracking physical activity data. JAMA. 2015;313(6):625-626.

4. Tudor-Locke C, Sisson SB, Collova T, Lee SM, Swan PD. Pedometerdetermined step count guidelines for classifying walking intensity in a young ostensibly healthy population. Can J Appl Physiol. 2005;30(6):666676.

5. Patel MS, Small DS, Harrison JD, et al. Effectiveness of behaviorally designed gamification interventions with social incentives for increasing physical activity among overweight and obese adults across the United States: the STEP UP randomized clinical trial. JAMA Intern Med. 2019; 179:1-9.

Publisher's Note: Springer Nature remains neutral with regard to jurisdictional claims in published maps and institutional affiliations. 